\title{
Different forms of adiponectin reduce the apoptotic and damaging effect of cigarette smoke extract on human bronchial epithelial cells
}

\author{
MENG-YU CHENG ${ }^{1}$, HU LIU $^{1}$, TIE-MEI ZHANG ${ }^{2}$ and JIAN-YING XU ${ }^{1}$ \\ ${ }^{1}$ Department of Respiratory Medicine, Dayi Hospital Affiliated to Shanxi Medical University, Taiyuan, Shanxi 030032; \\ ${ }^{2}$ Second Department of Respiratory Medicine, Jimo City People's Hospital, Qingdao, Shandong 266200, P.R. China
}

Received May 2, 2015; Accepted September 22, 2016

DOI: $10.3892 / \mathrm{etm} .2016 .3872$

\begin{abstract}
Chronic obstructive pulmonary disease (COPD) is a common respiratory disease, in which adiponectin may serve an important role. The present study investigated the role of adiponectin in the apoptotic and damaging effect of cigarette smoke extract (CSE) on human bronchial epithelial cells (16HBECs). An MTT assay showed that CSE significantly inhibited the proliferation of 16HBECs ( $\mathrm{F}=1808.88$, $\mathrm{P}<0.01)$. The 16HBECs were treated with different concentrations of high molecular weight (HMW) adiponectin and globular domain (gAd) adiponectin and it was observed that HMW and gAd dose-dependently inhibited the expression of tumor necrosis factor (TNF)- $\alpha$ and interleukin (IL)-8, and the generation of 4-hydroxy-nonenal and reactive oxygen species (ROS) in 16HBECs, thereby blocking the upregulating effect of CSE on these factors. However, the inhibitory effect of gAd on TNF- $\alpha$ and IL-8 expression was stronger compared with that of HMW, but the suppressing effect of HMW on ROS production was superior compared with that of gAd. Further testing of apoptosis indicated that CSE and HMW promoted the apoptosis of 16HBECs. However, such effects of HMW declined with an increase in concentration. In contrast, $\mathrm{gAd}$ showed an inhibitory effect on apoptosis and inhibited the occurrence of CSE-induced apoptosis in a dose-dependent manner. Therefore, the present study demonstrated that different forms of adiponectin may have different mechanisms of action, suggesting that further exploration of their effects may open a new avenue for the treatment of COPD.
\end{abstract}

Correspondence to: Dr Jian-Ying Xu, Department of Respiratory Medicine, Dayi Hospital Affiliated to Shanxi Medical University, 99 Longcheng Street, Taiyuan, Shanxi 030032, P.R. China E-mail: xujyty@126.com

Key words: adiponectin, cigarette smoke extract, chronic obstructive pulmonary disease, apoptosis

\section{Introduction}

COPD is a chronic inflammatory disease characterized by airflow limitation, with a high morbidity and mortality. According to previously reported statistics, COPD ranks fourth in the cause of mortality worldwide, and its rank is expected to move up to third in 2030 (1). The prevalence rate of COPD among people over age 40 is $8.2 \%$ in China (2). COPD has already become an important global public health problem. Therefore, further study and understanding of COPD are of great significance.

Epidemiological studies have shown that smoking is the most important pathogenic factor of human COPD (3). Long-term exposure to tobacco smoke can cause the infiltration and recruitment of lung inflammatory cells, which can then release various cytokines and finally cause the formation of COPD through a variety of mechanisms (4). Among COPD patients, $80-90 \%$ are smokers or ex-smokers $(5,6)$. Studies showed that $\sim 15-20 \%$ of smokers eventually develop COPD (7), and a survey in China indicated that the prevalence of COPD among smokers was 2-4 times of that among non-smokers, and was directly associated with the duration and quantity of smoking, i.e., a longer duration and a greater amount of smoking results in a higher prevalence of COPD (8).

Adiponectin is an adipokine secreted by adipocytes. Previously, a study identified that airway epithelial cells also secrete adiponectin, which through autocrine and paracrine mechanisms regulates chronic inflammation in COPD (9). Tomoda et al (10) used ELISA to detect the serum level of adiponectin in male patients with COPD and found that adiponectin levels in the serum of patients with COPD was significantly higher compared with that of a normal control, suggesting that COPD may be closely associated with adiponectin. Chan et al (11) studied the association between adiponectin, interleukin (IL)-6, IL-8 and C-reactive protein with COPD, and found that adiponectin, IL-6 and C-reactive protein were significantly elevated in patients with COPD. Miller et al (12) used an adiponectin null mouse model to study the association between adiponectin and emphysema and observed an increase in diseased animals. Daniele et al (13) used ELISA technique and demonstrated that in patients with COPD, adiponectin levels were significantly upregulated, 
suggesting the involvement of adiponectin in the occurrence and development of COPD.

Previous have shown that adiponectin serves an anti-inflammatory role in the occurrence and development of COPD. The study of Tilg and Moschen (14) indicated that in patients with COPD, the anti-inflammatory effect of adiponectin was primarily achieved through inhibiting the activity of macrophages and the release of pro-inflammatory cytokines, such as IL-6 and tumor necrosis factor (TNF)- $\alpha$, from macrophages. In addition, adiponectin was shown to reduce the proliferation of lymphocytes, reduce lymphocyte reactivity, and induce the production of anti-inflammatory cytokines, such as IL-10 and IL-1 receptor antagonist, in monocytes and macrophages (14). Wert (15) injected exogenous adiponectin into wild-type and adiponectin-deficient mice and induced the production of TNF- $\alpha$ in macrophages by lipopolysaccharide stimulation; it was observed that the quantity of TNF- $\alpha$ generation reduced in adiponectin-null mice compared with the control, suggesting the anti-inflammatory effect of adiponectin.

A previous demonstrated that adiponectin has a pro-inflammatory function in COPD. Miller et al (9) found that adiponectin can bind to the inducible adiponectin receptor 1 on airway epithelial cells and macrophages, promote the production of IL-8, TNF- $\alpha$ and other inflammatory mediators, and recruit the aggregation of various inflammatory cells (shch as macrophages and neutrophils) by chemotaxis. The synthesis and secretion of IL-8 further promotes the production of adiponectin and induces the synthesis of adiponectin receptor 1 on airway epithelial cells and macrophages, resulting in a cascade effect via autocrine and paracrine mechanisms and thereby exerting an anti-inflammatory effect. Thus, adiponectin serves dual anti- and pro-inflammatory roles in the occurrence and development of COPD; however, further investigation is required in order to determine which role exerts the leading effect. In the present study, cigarette smoke extract (CSE) stimulates human bronchial epithelial cells to simulate the occurrence of COPD, and the mechanism of adiponectin in this process is researched.

\section{Materials and methods}

Cell culture. Human bronchial epithelial cells (16HBECs) were purchased from ScienCell Research Laboratories, Inc. (Carlsbad, CA, USA), and cultured in Minumum Essential Medium (MEM; Gibco; Thermo Fisher Scientific, Inc., Waltham, MA, USA) supplemented with $10 \%$ fetal bovine serum (Gibco; Thermo Fisher Scientific, Inc.) at $37^{\circ} \mathrm{C}$ in a $5 \%$ $\mathrm{CO}_{2}$ incubator.

CSE preparation. CSE was prepared as described by Su et al (16) with modifications. Briefly, a stick of Da Qian Men cigarette (containing tar $0.013 \mathrm{~g}$, nicotine $0.001 \mathrm{~g}$ and CO 0.014 g; Taiyuan Tobacco Company, Taiyuan, China) was connected to a syringe driving instrument (Fig. 1A). The syringe was filled with $20 \mathrm{ml}$ serum-free MEM, and the cigarette was lit. To simulate human smoking, the sucking lasted $2 \mathrm{sec}$, and the pause was $58 \mathrm{sec}$, to fully absorb the smoke. Each cigarette took 7-8 min, and two cigarettes were tested continuously. The $20 \mathrm{ml}$ serum-free MEM were then prepared into CSE stock solution after being adjusted to $\mathrm{pH}$ 6.8-7.2 with
$1 \mathrm{~N} \mathrm{NaOH}$ (Amresco, Inc., Farmingham, MA, USA) and sterilized by filtering through a $0.22 \mu \mathrm{m}$ filter membrane (Merck Millipore, Shanghai, China). The CSE stock solution was then diluted with serum-free MEM into 0 (no CSE intervention group), $2.5,5,7.5$ and $10 \%$ culture media, which were then used in the experiment within $30 \mathrm{~min}$.

MTT assay. 16HBECs in the exponential growth were digested, resuspended, adjusted into $5 \times 10^{4}$ cells $/ \mathrm{ml}$, and seeded into 96-well plates at $200 \mu \mathrm{l} /$ well in triplicate. After the cells were grown into $70-80 \%$ confluence $24 \mathrm{~h}$ later and washed twice with D-Hank's solution (Gibco; Thermo Fisher Scientific, Inc.), each well was added $200 \mu \mathrm{l}$ of serum-free MEM containing 0, 2.5, 5.0, 7.5 and 10.0\% diluted CSE stock solution. After another $24 \mathrm{~h}$ of culture, the CSE medium in each well was replaced with fresh medium containing $20 \mu \mathrm{l}$ MTT (Amresco, Inc.) and $180 \mu \mathrm{l}$ MEM, and the cells were cultured for another $4 \mathrm{~h}$. The MTT medium was removed, and the plate was added $150 \mu \mathrm{l}$ DMSO/well and shaken for $10 \mathrm{~min}$. The absorbance was then measured on an automatic microplate reader (Bio-Rad 680; Bio-Rad Laboratories, Inc. Hercules, CA, USA) at $490 \mathrm{~nm}$ wavelength (A490). The inhibition rates were calculated based on the A490 values of the control (without CSE) and the blank (without CSE and MTT) wells. The CSE concentration with a cell inhibition rate of $50 \%$ was defined as CSE $\mathrm{IC}_{50}$ (inhibitory concentration of $50 \%$ ). Inhibition rate $=($ control well - experimental well $) /($ control well - blank well) x $100 \%$.

Detection of TNF- $\alpha, I L-8$ and 4-hydroxy-nonenal (HNE) with ELISA. The cells inoculated onto 6 -well plate at $1 \times 10^{5}$ cells $/ \mathrm{ml}$ were randomly divided into the following 8 groups: i) CSE group, $2 \mathrm{ml} \mathrm{MEM} /$ well containing $100 \mu \mathrm{l}$ of CSE (5\% CSE); ii) negative control group, equal volume of D-Hank's solution/well; iii) high molecular weight (HMW) groups, first incubated in medium containing 5, 10 and $20 \mu \mathrm{g} / \mathrm{ml} \mathrm{HMW}$ (Gibco; Thermo Fisher Scientific, Inc.) for $2 \mathrm{~h}$ at $37^{\circ} \mathrm{C}$, and then cultured in 5\% CSE medium; iv) globular domain (gAd) groups, firstly incubated in medium containing 5, 10 and $20 \mu \mathrm{g} / \mathrm{ml} \mathrm{gAd}$ (Gibco; Thermo Fisher Scientific, Inc.) for $2 \mathrm{~h}$ at $37^{\circ} \mathrm{C}$, and then cultured in 5\% CSE medium. The above groups were cultured for $24 \mathrm{~h}$, and the cells and supernatants were harvested and used for the subsequent experiments. The experiments were repeated three times, and the supernatants were collected to detect the contents of TNF- $\alpha$ (cat. no. EHC103a; Human TNF- $\alpha$ ELISA kit; Neobioscience, Shenzhen, China), IL-8 (cat. no. EHC008; Human IL-8 ELISA kit; Neobioscience), and 4-HNE (cat. no. E01H0203; Human 4-HNE ELISA kit; BlueGene Biotech Co., Ltd., Shanghai, China) proteins by ELISA.

Reactive oxygen species (ROS) determination. After $24 \mathrm{~h}$ of culture, the 6-well plate was removed from the incubator, and the cells were washed three times with preheated sterile D-Hank's buffer. The cells in each group were then added $1.5 \mathrm{ml}$ medium containing $10 \mu \mathrm{M}$ DCFH-DA (NKKCBio, Nanjing, China) and incubated for $40 \mathrm{~min}$ at $37^{\circ} \mathrm{C}$ in a $5 \% \mathrm{CO}_{2}$ incubator. After incubation, the cells were washed three times with D-Hank's solution to remove the extracellular fluorescent substances and reduce the background. The cells were then 
Table I. Injury of $5 \%$ CSE on $16 \mathrm{HBECs}( \pm$ standard deviation).

\begin{tabular}{lcccc}
\hline Group & TNF- $\alpha(\mathrm{pg} / \mathrm{ml})$ & $\mathrm{IL}-8(\mathrm{pg} / \mathrm{ml})$ & 4-HNE $(\mu \mathrm{g} / \mathrm{ml})$ & ROS (mean fluorescence intensity) \\
\hline Control & $8.43 \pm 2.85$ & $40.19 \pm 8.27$ & $0.16 \pm 0.13$ & $17.33 \pm 3.06$ \\
$5 \%$ CSE & $51.12 \pm 5.75$ & $306.43 \pm 27.09$ & $2.21 \pm 0.24$ & $346.52 \pm 13.71$ \\
T-value & -11.52 & -16.28 & -12.93 & -40.59 \\
P-value & $<0.01$ & $<0.01$ & $<0.01$ & $<0.01$ \\
\hline
\end{tabular}

CSE, cigarette smoke extrack; 4-HNE, 4-hydroxy-nonenal; IL-8, interleukin-8; ROS, reactive oxygen species; TNF- $\alpha$, tumor necrosis factor- $\alpha$.

A

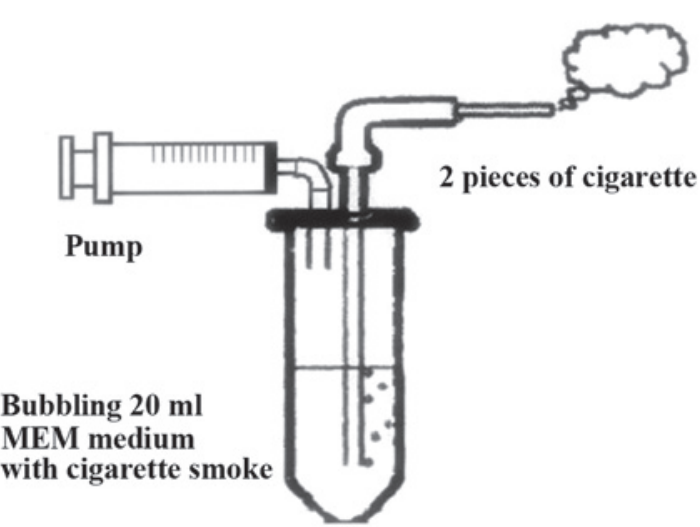

B

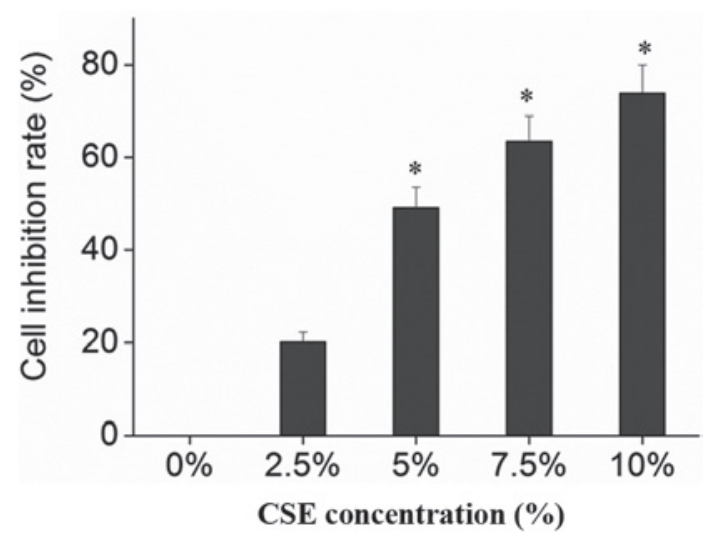

Figure 1. Inhibitory effect of CSE on 16HBECs. (A) The syringe driving instrument which was used to prepare CSE. (B) 16HBECs were treated with CSE in different concentrations, and MTT was used to detect cell inhibition rate. ${ }^{*} \mathrm{P}<0.05$ vs. 0\% CSE. CSE, cigarette smoke extract; MEM, Minumum Essential Medium.

collected and analyzed by flow cytometry (Beckman Coulter, Inc., Brea, CA, USA) and detected by Cytomics FC flow cytometry (Beckman Coulter, Inc.) within $1 \mathrm{~h}$.

Apoptosis. The cells were harvested by digestion with EDTA-free trypsin (Gibco; Thermo Fisher Scientific, Inc.) and washed twice with D-Hank's solution (by centrifugation at $200 \times \mathrm{g}$ for $5 \mathrm{~min}$ at room temperature). The cells $\left(1-5 \times 10^{5}\right)$ were then resuspended in $500 \mu \mathrm{l}$ binding buffer, mixed with $5 \mu \mathrm{l}$ annexin V-FITC (KenGen Biotech Co., Ltd., Nanjing, China) and $5 \mu$ l propidium iodide sequentially, incubated at room temperature in the dark for 5-15 min, and detected by flow cytometry (Beckman Coulter, Inc., Brea, CA, USA) within $1 \mathrm{~h}$.

Statistical analysis. All data were presented as the mean \pm standard deviation and statistically analyzed using SPSS version 17.0 software (SPSS, Inc., Chicago, IL, USA). The comparison among groups was performed using one-way analysis of variance, and the pairwise comparison between groups was conducted using Fisher's least significant difference t-test. $\mathrm{P}<0.05$ was considered to indicate a statistically significant difference.

\section{Results}

Inhibitory effect of CSE on 16HBECs. Bronchial epithelial cells are the first barrier to protect the airway tissue from the invasion of harmful substances, with a wide range of physiological functions. Bronchial epithelial cells are the major and first target cells to suffer from injuries in acute and chronic airway diseases. Upon activation by the stimuli of external harmful substances, they promote the expression of inflammatory cytokines and actively participate in the process of airway inflammation via secretion of inflammatory mediators, cytokines and growth factors (9). Therefore, the present study used 16HBECs as a model to investigate the effects of CSE and adiponectin. MTT assay indicated that CSE significantly inhibited the proliferation of 16HBECs $(\mathrm{F}=1808.88$, $\mathrm{P}<0.01)$ in a dose-dependent manner, and the $\mathrm{IC}_{50}$ of CSE on 16HBECs was $\sim 5 \%$ (Fig. 1B).

Adiponectin reverses the CSE-upregulated expression of TNF- $\alpha$ and IL- 8 in 16HBECs. TNF- $\alpha$ and IL- 8 serve important roles in the chronic airway inflammation of COPD. A previous study demonstrated that the levels of IL-8 and TNF- $\alpha$ are significantly elevated in patients with COPD compared with the normal control, and are significantly higher in the acute exacerbation phase compared with in the stable phase (17). They are important inflammatory markers in evaluating the condition and prognosis of COPD (18). ELISA detection of the secretion of TNF- $\alpha$ and IL- 8 from 16HBECs treated with 5\% CSE indicated significantly increased expression of TNF- $\alpha$ and IL-8 (Table I; P<0.01), suggesting that CSE may promote the expression of TNF- $\alpha$ and IL- 8 and thereby cause injury to $16 \mathrm{HBEC}$. In the serum, adiponectin proteins exist in the forms of full length structure (triploid, hexaploid and HMW) and gAd (14). The present study explored the specific 
A

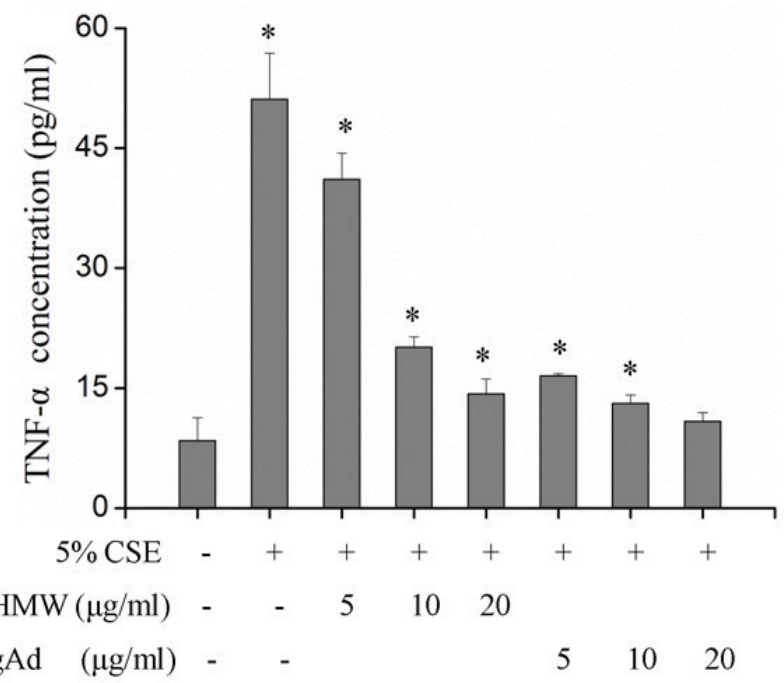

B

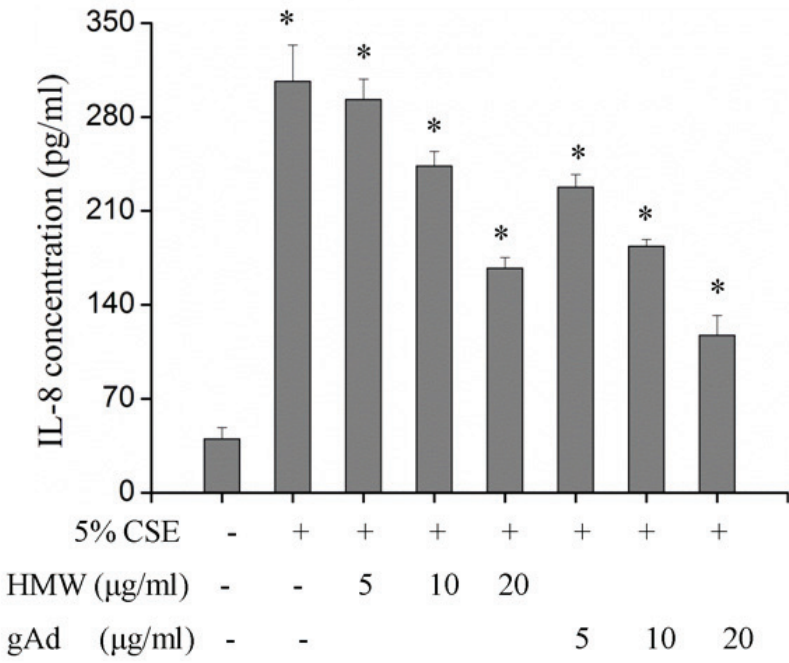

Figure 2. Adiponectin reversed CSE-upregulated expression of TNF- $\alpha$ and IL-8 in 16HBECs. 16HBECs were treated with 5\% CSE, 5\% CSE + HMW or gAd at different concentrations, and concentrations of (A) TNF- $\alpha$ and (B) IL- 8 were detected by ELISA. Each bar represents the mean \pm standard deviation from three samples. * $\mathrm{P}<0.05$ vs. 5\% CSE (-)/HMW (-)/gAD (-). CSE, cigarette smoke extract; gAd, globular domain; HMW, high molecular weight; IL-8, interleukin-8; TNF- $\alpha$, tumor necrosis factor- $\alpha$.

Table II. Correlation of HMW, gAd and TNF- $\alpha$, IL-8, 4-HNE, ROS.

\begin{tabular}{llcccc}
\hline Group & & TNF- $\alpha$ & IL-8 & 4-HNE & ROS \\
\hline \multirow{2}{*}{ HMW } & R-value & -0.919 & -0.958 & -0.930 & -0.931 \\
& P-value & $<0.01$ & $<0.01$ & $<0.01$ & $<0.01$ \\
gAd & R-value & -0.755 & -0.959 & -0.933 & -0.965 \\
& P-value & $<0.01$ & $<0.01$ & $<0.01$ & $<0.01$ \\
\hline
\end{tabular}

gAd, globular domain; HMW, high molecular weight; 4-HNE, 4-hydroxy-nonenal; IL-8, interleukin-8; ROS, reactive oxygen species; TNF- $\alpha$, tumor necrosis factor- $\alpha$.

functions of different forms of adiponectin. Treatment of 16HBECs with different concentrations of HMW and gAd found that both forms inhibited the expression of TNF- $\alpha$ and IL-8 in a dose-dependent manner and significantly blocked the CSE-induced upregulation of TNF- $\alpha$ and IL-8 (Fig. 2; Table II). However, the inhibitory effect of gAd was more evident; while $5 \mu \mathrm{g} / \mathrm{ml}$ gAd significantly inhibited the expression of TNF- $\alpha$ and IL-8, a significant effect of HMW was only observed when its concentration reached $10 \mu \mathrm{g} / \mathrm{mL}$ (Fig. 2). At the same concentration, gAd displayed a stronger inhibitory effect on the expression of TNF- $\alpha$ and IL- 8 compared with HMW (Fig. 2).

Adiponectin blocks the upregulating effect of CSE on 4-HNE and ROS levels in 16HBECs. Tobacco smoke can induce inflammatory cells to infiltrate into the lung tissue and produce ROS (19). An excessive production of ROS can cause peroxidation of cellular proteins, lipids, DNA and carbohydrates. As one of the most stable representative terminal products of lipid peroxidation under pulmonary oxidative stress, 4-HNE is more stable than ROS and can diffuse to and exert effects in distant tissues. The detection of 4-HNE and ROS indicated that both HMW and gAd inhibited the production of 4-HNE and ROS in a dose-dependent manner and significantly blocked the upregulation of 4-HNE and ROS by CSE $(\mathrm{P}<0.01$; Fig. 3, Table II). Although there was no significant difference between the inhibitory effects of HMW and gAd on 4-NHE production, the suppression of ROS by HMW was more effective compared with gAd. The inhibitory effects of 5 and $10 \mu \mathrm{g} / \mathrm{ml} \mathrm{HMW}$ were equivalent to those of 10 and $20 \mu \mathrm{g} / \mathrm{ml}$ gAd, respectively (Fig. 3).

Effects of CSE and adiponectin on the apoptosis of 16HBECs. CSE treatment significantly increased the apoptosis of 16HBEC $(\mathrm{P}<0.01$; Fig. 4), suggesting that CSE can promote apoptosis in these cells. Intervention with different concentrations of HMW found that HMW further aggravated apoptosis; however, with the increase in HMW concentration, its apoptosis-promoting effect declined (Fig. 4). Use of different concentrations of gAd for intervention showed that gAd dose-dependently inhibited CSE-induced apoptosis (Fig. 4). These results indicate that different forms of adiponectin may have different activities, and that gAd has an anti-apoptotic effect.

\section{Discussion}

COPD is a common disease threatening public health worldwide. With a trend of increasing incidence, it has currently become the fourth biggest cause mortality worldwide (20). Present therapies cannot block the disease progression of COPD patients, which severely affects the treatment efficacy and prognosis of COPD (21). Therefore, further study on the pathogenesis and risk factors of COPD may provide a better theoretical basis for the prevention and treatment of COPD.

Previous studies have shown that cytokines serve important roles in the pathogenesis of COPD; among them, adiponectin has drawn increasing attention $(17,19)$. Adiponectin serves an important role in the body's metabolic processes; it has multiple biological functions, such as regulating lipid metabolism, 

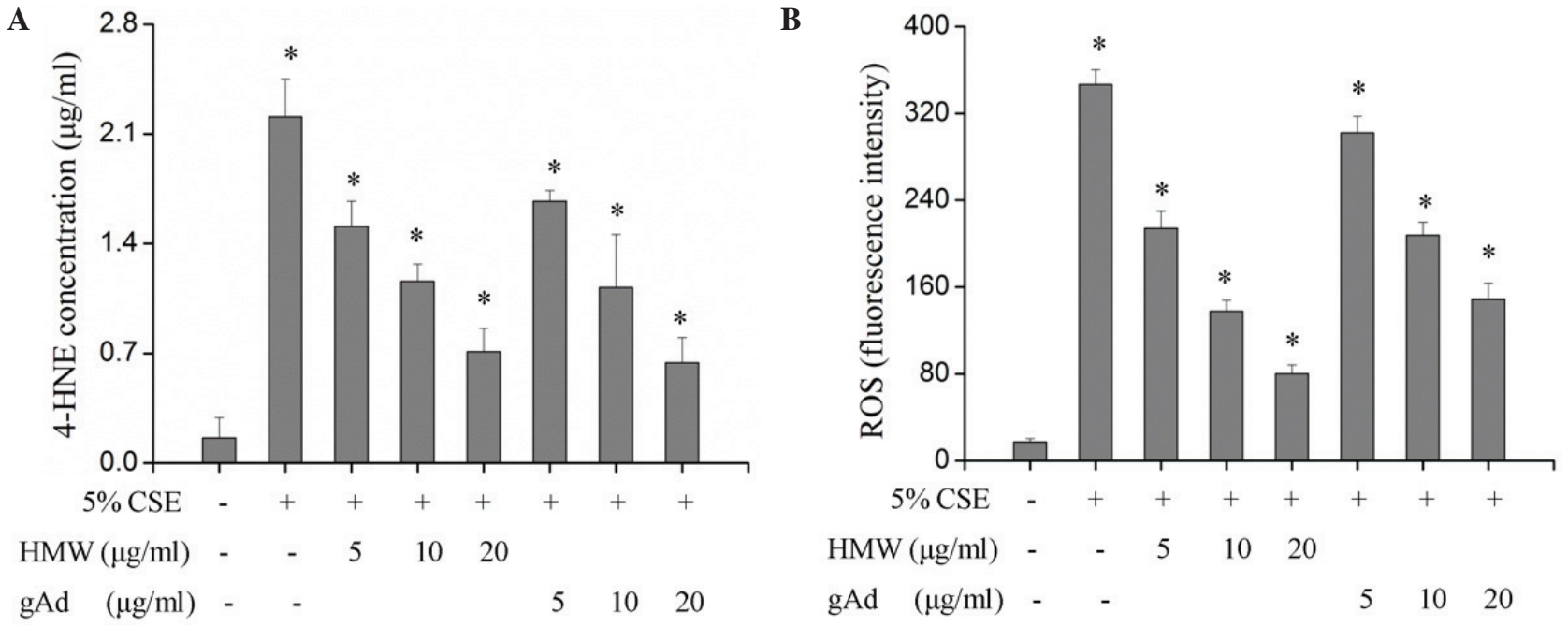

Figure 3. Adiponectin blocked the upregulating effect of CSE on 4-HNE and ROS levels in 16HBECs. 16HBECs were treated with 5\% CSE, 5\% CSE + HMW or gAd at different concentrations, and (A) 4-HNE was detected by ELISA and (B) ROS was detected by flow cytometry. Each bar represents the mean \pm standard deviation from three samples. "P<0.05 vs. 5\% CSE (-)/HMW (-)/gAD (-).CSE, cigarette smoke extract; gAd, globular domain; HMW, high molecular weight; 4-HNE, 4-hydroxy-nonenal; ROS, reactive oxygen species.

A
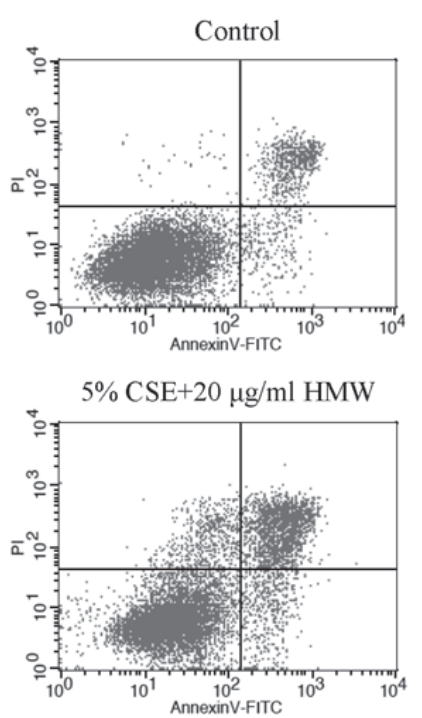

B
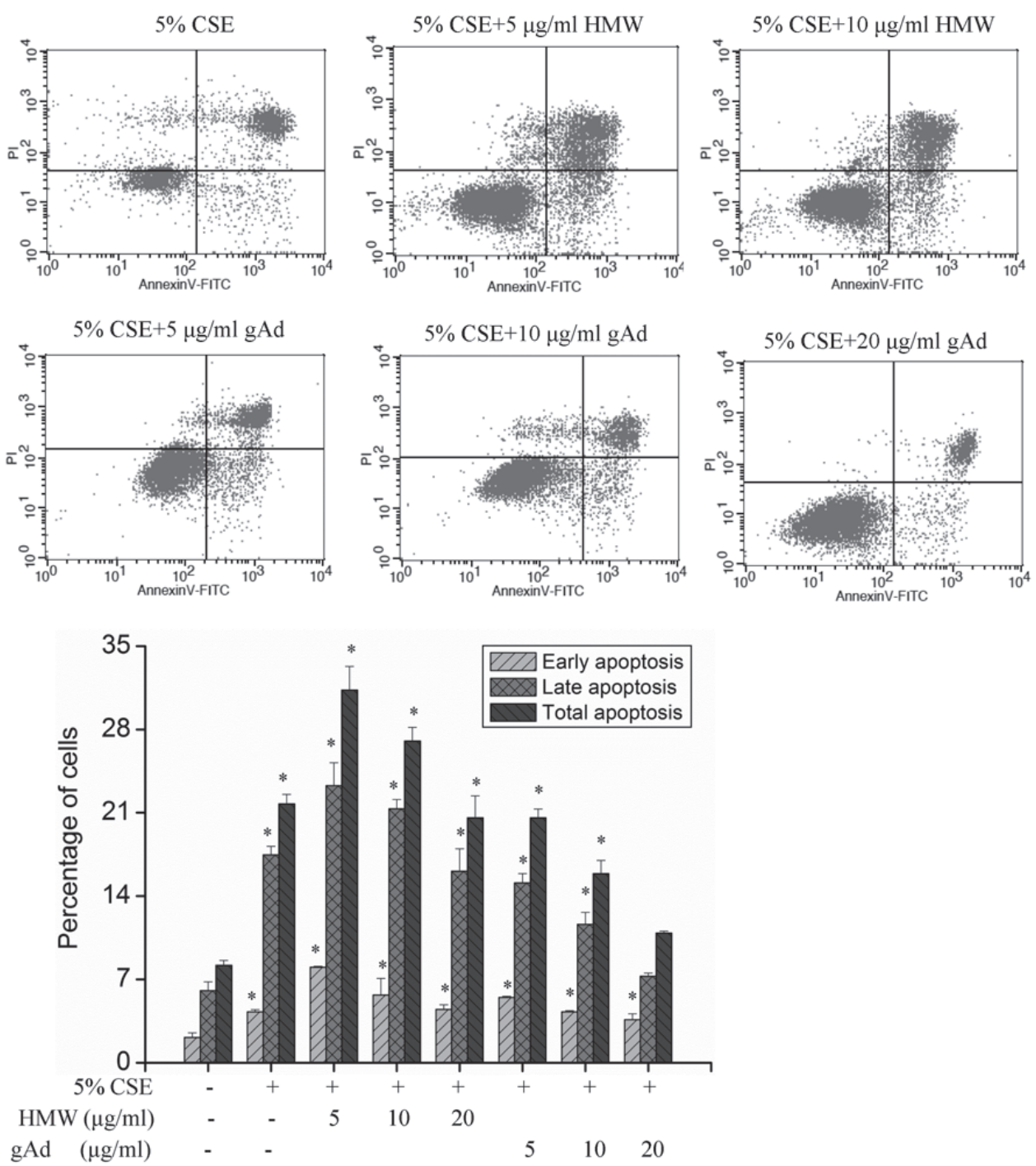

Figure 4. Effects of CSE and adiponectin on the apoptosis of 16HBECs. (A) 16HBECs were treated with 5\% CSE, 5\% CSE + HMW or gAd at different concentrations, and apoptosis was detected. (B) Quantitative analysis of apoptotic cell death induced by CSE or/and adiponectin in 16HBECs as measured by Annexin V-FITC-PI co-staining flow cytometric analysis. Results are presented as the mean ( $\mathrm{n}=3) \pm$ standard deviation. " $\mathrm{P}<0.05$ vs. $5 \%$ CSE (-)/HMW (-)/gAD (-). CSE, cigarette smoke extract; FITC, fluorescein; gAd, glocular domain; HMW, high molecular weight; PI, propidium iodide. 
improving insulin resistance, protecting the cardiovascular system, regulating bone formation, suppressing inflammation and tumor growth (22). In addition, a previous study demonstrated that adiponectin is associated with the pathophysiology of airway inflammation (23). Sull et al (24) studied 2,500 healthy male Koreans and found that smoking reduced human serum adiponectin levels, and that such reduction was significantly associated with the quantity of cigarettes smoked. Kirdar et al (25) showed that the serum levels of adiponectin, erythrocyte sedimentation rate and $\mathrm{C}$-reactive protein in the acute exacerbation phase of COPD were significantly higher compared with those in the stable phase, and hence believed that adiponectin may be a marker of systemic inflammation in COPD.

A previous study demonstrated that adiponectin serves a proinflammatory role in the pathogenesis of COPD (9). However, further investigation indicated that adiponectin exerted anti-inflammatory effects in COPD. Summer et al (26) studied a mouse model and confirmed that adiponectin was present in the lungs and exerted an anti-inflammatory effect via reducing the release of TNF- $\alpha$ and matrix metalloproteinase 12 through inhibiting the activation of alveolar macrophages; adiponectin deficiency or insufficiency changed the type of pulmonary emphysema in mice. Nakanishi et al (27) reported that adiponectin deficiency led to the occurrence of COPD in mice, accompanied by systemic inflammation and extrapulmonary effects; they also found that hypoadiponectinemia is associated with the loss of function of endothelial cells and may therefore serve a key role in the progression of COPD and concomitant complications. Yoon et al (28) reported a seven year cohort study which indicated that an increase of serum adiponectin levels reduced the level of mortality of patients with COPD from cardiovascular diseases. Therefore, the function of adiponectin needs to be further verified in future investigations.

The present study on $16 \mathrm{HBEC}$ cells revealed that both HMW and gAd dose-dependently inhibit the expression of TNF- $\alpha$ and IL-8 as well as the generation of 4-HNE and ROS; they also significantly block the upregulating effect of CSE on the above factors. TNF- $\alpha$ is an important inflammatory and immune-regulatory factor in the pathogenesis of COPD. A previous study demonstrated that $\mathrm{TNF}-\alpha$ serves a key role in tobacco smoke-induced chronic airway inflammation and connective tissue injury (29). The primary biological function of IL-8 is chemotactic effect, which is the major cause of the increase of pulmonary neutrophils in patients with COPD. Meanwhile, IL-8 is an important indicator used to assess the severity of chronic airway inflammation (30). Adiponectin can antagonize the upregulating effect of CSE on TNF- $\alpha$ and IL-8, indicating that adiponectin has an anti-inflammatory effect. In addition, oxidative stress serves a key role in the development and progression of COPD. Oxidative stress generates an excessive quantity of ROS, which may cause pathophysiological changes through various pathways (31). Lipid peroxidation products from ROS, such as 4-HNE, may serve as signal molecules to activate nuclear factor $-\kappa \mathrm{B}$, causing pulmonary and systemic inflammation in patients with COPD; these inflammatory responses generate more oxidative-stress products, forming a vicious cycle (32).

A previous study showed that the expression of 4-HNE significantly increased in mouse bronchial epithelial cells and type II alveolar epithelial cells upon exposure to tobacco smoke (33), suggesting that 4-HNE may be involved in the injuries to bronchial mucosa caused by smoking. The inhibitory effect of adiponectin on 4-HNE and ROS production in the present study demonstrates its anti-inflammatory function. However, it was observed that gAd exerted a stronger inhibitory effect on the expression of TNF- $\alpha$ and IL- 8 compared with HMW, while HMW more significantly inhibited the generation of ROS compared with gAd, suggesting that different forms of adiponectin may have different activities.

In the current study, analysis of apoptosis found that both CSE and HNW promoted apoptosis in 16HBECs, but interestingly, with an increase in concentration, the pro-apoptotic effect of HMW weakened. In contrast, gAd exerted an anti-apoptotic effect and dose-dependently inhibited the occurrence of CSE-induced apoptosis. These data further demonstrate that different forms adiponectin may have different mechanisms of action. Previous investigations have shown that HMW serves an anti-inflammatory role in monocyte-macrophages and vascular endothelial cells $(34,35)$, while gAd can induce macrophage apoptosis (36). However, it can be suggested that gAd has a role in the resistance of vascular endothelial cells to apoptosis and the protection of vascular endothelial cells, which is consistent with the findings of the present study. For example, a previous study demonstrated that gAd intervention of vascular endothelial cells increased the level of endothelial type nitric oxide (NO) synthase and thereby increased the production of NO, exerting a protective effect on vascular endothelial cells and serving an anti-atherosclerotic role (37). Zhao et al (38) reported that gAd significantly reduced apoptosis in vascular endothelial cells induced by persistent or fluctuating high glucose.

In conclusion, the present study explored the effects of HMW and gAd, two different forms of adiponectin, on CSE-induced apoptosis and injury in human bronchial epithelial cells, and found that both HMW and gAd dose-dependently reversed the upregulating effect of CSE on the levels of TNF- $\alpha$, IL-8, 4-HNE and ROS. In addition, gAd inhibited CSE-induced cell apoptosis. These results suggest that adiponectin may form a novel therapeutic target of COPD; however, in-depth studies with other lung parenchyma cells and animal models are required.

\section{Acknowledgements}

The present study was supported by the Natural Science Foundation of ShanXi Province (grant no. 2012011037-1) and the Youth Science and Technology Research Foundation of ShanXi Province (grant no. 2014021040-4).

\section{References}

1. Smolonska J, Wijmenga C, Postma DS and Boezen HM: Meta-analyses on suspected chronic obstructive pulmonary disease genes: A summary of 20 years' research. Am J Respir Crit Care Med 180: 618-631, 2009

2. Zhong N, Wang C, Yao W, Chen P, Kang J, Huang S, Chen B, Wang C, Ni D, Zhou Y, et al: Prevalence of chronic obstructive pulmonary disease in China: A large, population-based survey. Am J Respir Crit Care Med 176: 753-760, 2007.

3. Decramer M, Janssens W and Miravitlles M: Chronic obstructive pulmonary disease. Lancet 379: 1341-1351, 2012. 
4. Muro S: Cigarette smoking is the most important causal factor for developing chronic obstructive pulmonary disease (COPD). Nihon Rinsho 69: 1735-1740, 2011 (In Japanese).

5. Løkke A, Lange P, Scharling H, Fabricius P and Vestbo J: Developing COPD: A 25 year follow up study of the general population. Thorax 61: 935-939, 2006.

6. Bush A: COPD: A pediatric disease. COPD 5: 53-67, 2008.

7. Viegi G, Pistelli F, Sherrill DL, Maio S, Baldacci S and Carrozzi L: Definition, epidemiology and natural history of COPD. Eur Respir J 30: 993-1013, 2007.

8. Zhou Y, Wang C, Yao W, Chen P, Kang J, Huang S, Chen B, Wang C, Ni D, Wang X, et al: COPD in Chinese nonsmokers. Eur Respir J 33: 509-518, 2009.

9. Miller M, Cho JY, Pham A, Ramsdell J and Broide DH: Adiponectin and functional adiponectin receptor 1 are expressed by airway epithelial cells in chronic obstructive pulmonary disease. J Immunol 182: 684-691, 2009.

10. Tomoda K, Yoshikawa M, Itoh T, Tamak S, Fukuoka A, Komeda $\mathrm{K}$ and Kimura H: Elevated circulating plasma adiponectin in underweight patients with COPD. Chest 132: 135-140, 2007.

11. Chan KH, Yeung SC, Yao TJ, Ip MS, Cheung AH, Chan-Yeung MM and Mak JC; COPD Study Group of the Hong Kong Thoracic Society: Elevated plasma adiponectin levels in patients with chronic obstructive pulmonary disease. Int J Tuberc Lung Dis 14: 1193-1200, 2010.

12. Miller M, Pham A, Cho JY, Rosenthal P and Broide DH: Adiponectin-deficient mice are protected against tobacco-induced inflammation and increased emphysema. Am J Physiol Lung Cell Mol Physiol 299: L834-L842, 2010.

13. Daniele A, De Rosa A, Nigro E, Scudiero O, Capasso M, Masullo M, de Laurentiis G, Oriani G, Sofia M and Bianco A: Adiponectin oligomerization state and adiponectin receptors airway expression in chronic obstructive pulmonary disease. In J Biochem Cell Biol 44: 563-569, 2012.

14. Tilg H and Moschen AR: Adipocytokines: Mediators linking adipose tissue, inflammation and immunity. Nat Rev Immunol 6: 772-783, 2006.

15. Wert SE: Does adiponectin play a role in pulmonary emphysema? Am J Physiol Lung Cell Mol Physiol 294: L1032-L1034, 2008.

16. Su Y,Han W, Giraldo C, De Li Y and Block ER: Effect of cigarette smoke extract on nitric oxide synthase in pulmonary artery endothelial cells. Am J Respir Cell Mol Biol 19: 819-825, 1998.

17. Damiá Ade D, Gimeno JC, Ferrer MJ, Fabregas ML, Folch PA and Paya JM: A study of the effect of proinflammatory cytokines on the epithelial cells of smokers, with or without COPD. Arch Bronconeumol 47: 447-453, 2011.

18. Garrod R, Marshall J, Barley E, Fredericks S and Hagan G: The relationship between inflammatory markers and disability in chronic obstructive pulmonary disease (COPD). Prim Care Respir J 16: 236-240, 2007.

19. Kode A, Yang SR and Rahman I: Differential effects of cigarette smoke on oxidative stress and proinflammatory cytokine release in primary human airway epithelial cells and in a variety of transformed alveolar epithelial cells. Respir Res 7: 132, 2006.

20. Chorostowska-Wynimko J: The role of inflammation in the pathogenesis of chronic obstructive pulmonary disease. Pol Merkur Lekarski 17: 203-207, 2004 (In Polish).

21. Barnes PJ, Shapiro SD and Pauwels RA: Chronic obstructive pulmonary disease: Molecular and cellular mechanisms. Eur Respir J 22: 672-688, 2003.

22. Siasos G, Tousoulis D, Kollia C, Oikonomou E, Siasou Z, Stefanadis C and Papavassiliou AG: Adiponectin and cardiovascular disease: Mechanisms and new therapeutic approaches. Curr Med Chem 19: 1193-1209, 2012.
23. Bianco A, Mazzarella G, Tu'rchiarelli V, Nigro E, Corbi G, Scudiero O, Sofia M and Daniele A: Adiponectin: An attractive marker for metabolic disorders in Chronic Obstructive Pulmonary Disease (COPD). Nutrients 5: 4115-4125, 2013.

24. Sull JW, Kim HJ, Yun JE, Park EJ, Kim G and Jee SH: Serum adiponectin is associated with smoking status in healthy Korean men. Endocr J 56: 73-78, 2009.

25. Kirdar S, Serter M, Ceylan E, Sener AG, Kavak T and Karadağ F: Adiponectin as a biomarker of systemic inflammatory response in smoker patients with stable and exacerbation phases of chronic obstructive pulmonary disease. Scand J Clin Lab Invest 69: 219-224, 2009.

26. Summer R, Little FF, Ouchi N, Takemura Y, Aprahamian T, Dwyer D, Fitzsimmons K, Suki B, Parameswaran H, Fine A and Walsh K: Alveolar macrophage activation and an emphysema-like phenotype in adiponectin-deficient mice. Am J Physiol Lung Cell Mol Physiol 294: L1035-L1042, 2008.

27. Nakanishi K, Takeda Y, Tetsumoto S, Iwasaki T, Tsujino K, Kuhara H, Jin Y, Nagatomo I, Kida H, Goya S, et al: Involvement of endothelial apoptosis underlying chronic obstructive pulmonary disease-like phenotype in adiponectin-null mice: Implications for therapy. Am J Respir Crit Care Med 183: 1164-1175, 2011.

28. Yoon HI, Li Y, Man SF, Tashkin D, Wise RA, Connett JE, Anthonisen NA, Churg A, Wright JL and Sin DD: The complex relationship of serum adiponectin to COPD outcomes COPD and adiponectin. Chest 142: 893-899, 2012.

29. Chung KF: Cytokines as targets in chronic obstructive pulmonary disease. Curr Drug Targets 7: 675-681, 2006.

30. Celik H, Akpinar S, Karabulut H, Oktar P, Dursun B, Erguden HC, Gunay S and Sipit T: Evaluation of IL-8 nasal lavage levels and the effects of nasal involvement on disease severity in patients with stable chronic obstructive pulmonary disease. Inflammation 38: 616-622, 2015.

31. Antus B and Kardos Z: Oxidative stress in COPD: Molecular background and clinical monitoring. Curr Med Chem 22: 627-650, 2015.

32. MacNee W: Pulmonary and systemic oxidant/antioxidant imbalance in chronic obstructive pulmonary disease. Proc Am Thorac Soc 2: 50-60, 2005.

33. Aoshiba K, Koinuma M, Yokohori N and Nagai A: Immunohistochemical evaluation of oxidative stress in murine lungs after cigarette smoke exposure. Inhal Toxicol 15: 1029-1038, 2003.

34. Kobayashi H, Ouchi N, Kihara S, Walsh K, Kumada M, Abe Y, Funahashi T and Matsuzawa Y: Selective suppression of endothelial cell apoptosis by the high molecular weight form of adiponectin. Circ Res 94: e27-e31, 2004.

35. Pang TT and Narendran P: The distribution of adiponectin receptors on human peripheral blood mononuclear cells. Ann N Y Acad Sci 1150: 143-145, 2008.

36. Akifusa S, Kamio N, Shimazaki Y, Yamaguchi N and Yamashita Y: Regulation of globular adiponectin-induced apoptosis by reactive oxygen/nitrogen species in RAW264 macrophages. Free Radic Biol Med 45: 1326-1339, 2008.

37. Hattori Y, Suzuki M, Hattori S and Kasai K: Globular adiponectin upregulates nitric oxide production in vascular endothelial cells. Diabetologia 46: 1543-1549, 2003.

38. Zhao HY, Zhao M, Yi TN and Zhang J: Globular adiponectin protects human umbilical vein endothelial cells against apoptosis through adiponectin receptor 1/adenosine monophosphate-activated protein kinase pathway. Chin Med J (Engl) 124: 2540-2547, 2011. 